\title{
Splenic infarction in alcoholic hepatitis
}

\author{
Muaz Aijazi, MDª ${ }^{\mathrm{D}}$, Brendan Gill, $\mathrm{MD}^{\mathrm{a}} \mathbb{D}$, Arjun Kalaria, $\mathrm{MD}^{\mathrm{a}}$, and Ashish Ahuja, MD \\ ${ }^{a}$ Department of General Internal Medicine, University of Pittsburgh Medical Center Mercy Hospital, Pittsburgh, Pennsylvania; ${ }^{b}$ Department of \\ General Internal Medicine, University of Maryland, Baltimore, Maryland
}

\section{ABSTRACT}

Splenic infarctions have been reported in cirrhotic patients with comorbidities (e.g., primary biliary cirrhosis), but there is little to no mention of spontaneous splenic infarction in alcoholic hepatitis. This condition is notable, as splenic function has been shown to decrease in alcoholic liver disease, but the exact pathophysiology has been unclear. We describe a relatively young cirrhotic woman who developed splenic infarctions in the setting of alcoholic hepatitis.

KEYWORDS Acute hepatitis; alcoholic liver disease; cirrhosis; splenic infarction

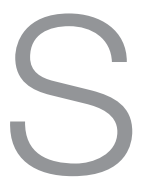

plenic infarction is generally described as either having a cardiovascular etiology or arising in hypercoagulable states. To date, only case reports exist describing splenic infarctions in patients with cirrhosis. In this report, we describe a young cirrhotic woman presenting with alcoholic hepatitis who was subsequently found to have splenic infarctions.

\section{CASE DESCRIPTION}

A 40-year-old white woman with known hepatic steatosis secondary to alcohol abuse and irritable bowel syndrome presented to our hospital with worsening jaundice, nausea, and vomiting. Her alanine aminotransferase was $54 \mathrm{U} / \mathrm{L}$; aspartate transaminase, $246 \mathrm{U} / \mathrm{L}$; alkaline phosphatase, $589 \mathrm{U} / \mathrm{L}$; total bilirubin, $14.0 \mathrm{mg} / \mathrm{dL}$; international normalized ratio, 1.9; and ethanol level, $240 \mathrm{mg} / \mathrm{dL}$. Her white blood cell count was $20,900 / \mu \mathrm{L}$ with $92 \%$ neutrophils. At this point, despite a Maddrey score $>50$, we decided to forego corticosteroids given her infection. After a 5-day course of intravenous ceftriaxone, however, her white blood cell count decreased to $18,400 / \mu \mathrm{L}$.

Because of the patient's worsening abdominal symptoms after a course of antibiotics, a right upper quadrant ultrasound was done, which showed only hepatic steatosis. Computed tomography with contrast showed cirrhosis with progressive moderate hepatomegaly as well as borderline splenomegaly with mild ascites (Figure 1a). She began to have small amounts of tarry stool, and an esophagogastroduodenoscopy yielded nothing of clinical interest. No infectious process was discovered even as abdominal pain, leukocytosis, and total bilirubin levels continued to worsen. Antinuclear antibody, Gliadin IgA, tissue transglutaminase, human immunodeficiency virus, and hepatitis panels were negative. Blood cultures remained negative, but our infectious diseases service recommended treating her with empiric meropenem.

With her severe abdominal pain and white blood cell count $>50,000 / \mu \mathrm{L}$ even after a 7 -day course of meropenem, a contrast CT scan was repeated (10 days after initial CT imaging). At this time, lesions in the spleen concerning for infarction were seen (Figure 1b). The decision was made to manage her conservatively, and her condition improved until discharge. Upon discharge, her white blood cell count was $13,000 / \mu \mathrm{L}$. Her abdominal pain and nausea had resolved to a comfortable level. Review of past electrocardiograms showed normal sinus rhythm. A CT scan 6 weeks after discharge showed resolution of these infarctions.

\section{DISCUSSION}

In a patient who presented with alcoholic hepatitis, with a CT scan showing cirrhotic morphology, we have described splenic infarctions that had not been noted on previous imaging. She was ultimately able to be managed conservatively. A mere handful of case reports exist describing splenic infarctions in cirrhotic patients with portal hypertension. In fact, of 152 patients with splenic infarctions in one study, only 3 cases were found to be secondary to cirrhosis or portal 

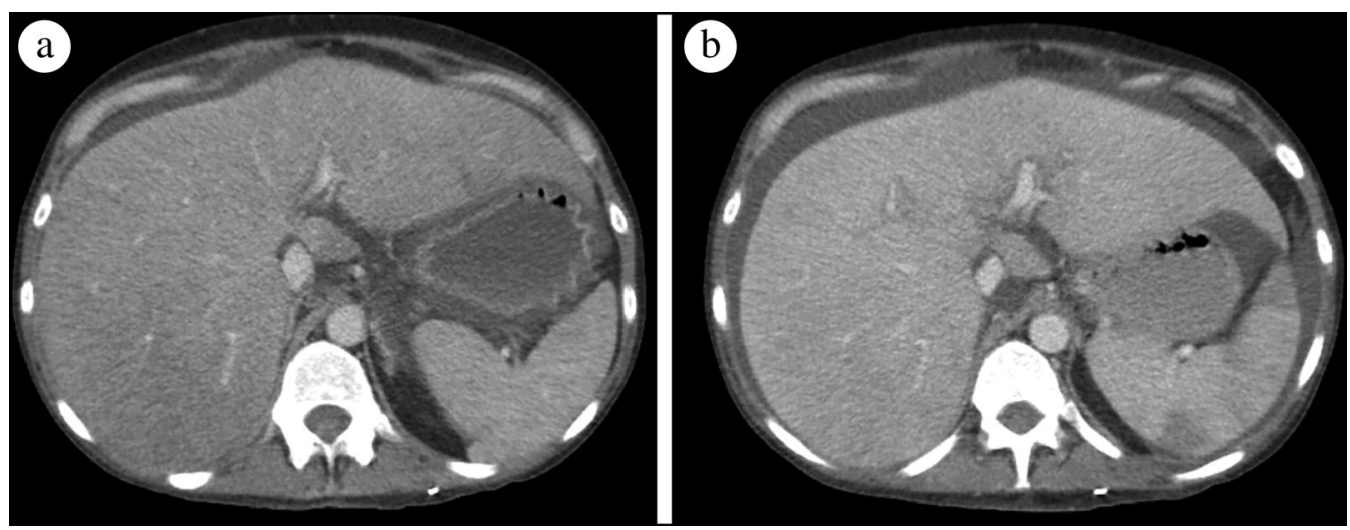

Figure 1. (a) Initial CT scan. (b) CT scan 10 days later showing splenic infarctions.

hypertension. ${ }^{1}$ Most patients $\leq 40$ years had an associated hematologic disorder, while those $>41$ years often had an embolic event. Left upper quadrant abdominal pain was the most common complaint in all patients, regardless of age or etiology. Leukocytosis was present in $49 \%$ of patients.

It is currently hypothesized that splenic infarctions in cirrhosis are due more to hemodynamic changes than organic occlusion of splenic vasculature. Splenomegaly results from portal hypertension, and this is believed to increase splenic oxygen requirements disproportionate to the body's oxygendelivering capacity. Consequently, the spleen suffers anoxic injury and subsequent infarction. ${ }^{2}$

The acuity of splenic infarction in our patient is notable, developing at some point in the 10 days between her CT scans. Judging by the timeline of her imaging and clinical picture, we believe the burden of her alcoholic hepatitis coupled with her urinary tract infection and then melena further compromised her oxygen-delivering capacity and she developed these infarctions. The nature of resolution of these infarcts is also notable; in one case report of splenic infarction in a cirrhotic patient, spontaneous resolution was not seen, even after 6 months. ${ }^{2}$ Spontaneous resolution of splenic infarction has, however, been described in patients with cardioembolic splenic infarctions. ${ }^{3,4}$

The patient's prolonged leukocytosis without evidence of active infection is likely related more to leukemoid reac- tion than splenic infarction. Mitchell et al found that patients with alcoholic hepatitis who developed leukemoid reactions generally had white blood cell counts $>40,000 /$ $\mu \mathrm{L}$, and the average was about $73,000 / \mu \mathrm{L}{ }^{5}$ They noted a remarkably high mortality rate, with patients dying a mean of 32 days after admission, regardless of Maddrey score. ${ }^{5}$ Our patient was able to be discharged home after symptom resolution.

\section{ORCID}

Muaz Aijazi iD http://orcid.org/0000-0001-7481-2341

Brendan Gill (ID) http://orcid.org/0000-0003-1629-8895

1. Jaroch MT, Broughan TA, Hermann RE. The natural history of splenic infarction. Surgery. 1986;100(4):743-750.

2. Chin JK, McCormick PA, Hilson AJ, Burroughs AK, McIntyre N. Liver/spleen scintigraphy for diagnosis of splenic infarction in cirrhotic patients. Postgrad Med J. 1993;69(815):715-717. doi:10.1136/pgmj. 69.815.715.

3. Vagenakis AG, Abreau CM, Braverman LE. Splenic infarction diagnosed by photoscanning. J Nucl Med. 1972;13(7):563-564.

4. Spencer RP. Healing of a splenic infarct. J Nucl Med. 1974;15(4): 303-304.

5. Mitchell RG, Michael M 3rd, Sandidge D. High mortality among patients with the leukemoid reaction and alcoholic hepatitis. South Med J. 1991;84(2):281-282. doi:10.1097/00007611-199102000-00036. 